\title{
A Real-Time PCR for Detection and Quantification of Mycoplasma ovipneumoniae
}

\author{
Falong YANG ${ }^{1}$, Xiaofang DAO ${ }^{1}$, Alex RODRIGUEZ-PALACIOS ${ }^{2)}$, Xufei FENG $^{1)}$, Cheng TANG ${ }^{1)}$, Xiaonong YANG ${ }^{1)}$ \\ and Hua $\mathrm{YUE}^{1) *}$ \\ ${ }^{1)}$ Department of Veterinary Medicine, College of Life Science and Technology, Southwest University for Nationalities, Chengdu 610041, \\ China \\ ${ }^{2)}$ Division of Gastroenterology and Liver Disease, Case Western Reserve University, Cleveland, OH 44106, U.S.A.
}

(Received 22 February 2014/Accepted 7 August 2014/Published online in J-STAGE 26 August 2014)

ABSTRACT. A real-time PCR for detection and quantification of $M$. ovipneumoniae was developed using 9 recently sequenced $M$. ovipneumoniae genomes and primers targeting a putative adhesin gene $p 113$. The assay proved to be specific and sensitive (with a detection limit of 22 genomic DNA) and could quantify M. ovipneumoniae DNA over a wide linear range, from $2.2 \times 10^{2}$ to $2.2 \times 10^{7}$ genomes. KEY WORDS: Mycoplasma ovipneumoniae, quantification, real-time PCR

doi: 10.1292/jvms.14-0094; J. Vet. Med. Sci. 76(12): 1631-1634, 2014

Mycoplasma ovipneumoniae has been associated with non-progressive pneumonia in sheep, goats and some wild small ruminants $[2,4,13]$. While $M$. ovipneumoniae can cause pneumoniae in its own right, it can predispose the hosts to invasion by other pathogens, such as Mannheimia haemolytica [4], which may enhance the pathological process.

Traditional microbiological culture techniques for isolation and identification of M. ovipneumoniae are cumbersome and usually can take up to 2 weeks for completion due to slow growth rate and the fastidious nutritional requirements of M. ovipneumoniae. Culture-based diagnosis is thus rarely undertaken routinely, which is often performed at highly specialized laboratories. Although conventional PCR [11] for M. ovipneumoniae detection has been developed and widely used in diagnosis and epidemiological investigation of $M$. ovipneumoniae infection [2, 4, 12, 13], tradictional PCR results are merely binary and can not quantify $M$. ovipneumoniae load in samples. Traditional culture techniques for Mycoplasma spp, such as plate-counting method, can also be quantitative; however, mycoplasmas grow slowly in culture medium yielding very small colonies after one week of incubation. Validated diagnostic assays for accurate and rapid quantification of $M$. ovipneumoniae are needed.

Real-time quantitative PCR (qPCR) is a reliable method for quantification of both DNA and RNA molecules in samples and is often more sensitive to conventional PCR assays. Recently, a number of real-time PCR assays have been described for a variety of mycoplasmas [1, 3, 6, 7, 9, 10]. Here, we reported the development and validation of a

*Correspondence to: Yue, H., Department of Veterinary Medicine, College of Life Science and Technology, Southwest University for Nationalities, Chengdu 610041, China.

e-mail: yhua900@hotmail.com

C2014 The Japanese Society of Veterinary Science

This is an open-access article distributed under the terms of the Creative Commons Attribution Non-Commercial No Derivatives (by-nc-nd) License $<$ http://creativecommons.org/licenses/by-nc-nd/3.0/>.
SYBR Green based qPCR assay specific and sensitive for $M$. ovipneumoniae DNA.

The first step in developing the qPCR was to identify genes common to referent $M$. ovipneumoniae strains and absent in other mycoplasmas. As we previously sequenced the genomes of $9 \mathrm{M}$. ovipneumoniae strains, including the field strain SC01 [16], type strain Y-98 and other 7 isolates (Table 1), we first identified orthologous genes among those 9 fully sequenced genomes using OrthoMCL version 2.0 [8]. Then, we used MUSCLE v3.7 [5] to perform a multiple sequence alignment of conserved genes. BLAST was then use to exclude genes with high homology with genes present in other microorganisms. Subsequently, a manual screening was performed to select genes with highly conserved regions among the 9 strains and further tested them as targets for subsequent primer design using Beacon designer 7.0 (PREMIER Biosoft International, Palo Alto, CA, U.S.A.). BLAST was then used to exclude primers with high homology for other microorganisms, including mycoplasmas. The sequence that was specific for $M$. ovipneumoniae in silico and that had the highest score attributed by the software was chosen for primer development for the real-time PCR. The primers that were deemed appropriate and finally selected for conducting real-time PCR were P113F (5'-TCTCCCAGATGATGCTAACC- $3^{\prime}$ ) and P113R (5'-TGAAAATCAACTGGTCTAA-3'), which amplifies a 295bp fragment of the $p 113$ gene coding for a putative adhesin P113 [17].

The bacterial strains used as referents to test the performance of the real-time PCR primers and the protocol, are listed in Table 2. Mycoplasmas were propagated in Hayflick broth containing $20 \%$ horse serum, whereas other bacterial strains were grown in Trypticase Soy broth. Clinical samples were also collected from animals that exhibited symptoms, such as coughing, nasal discharge and dyspnea, to test the performance of the qPCR. Clinical samples included 98 nasal swabs and 28 lung tissue samples collected from 4 goat herds from the Sichuan province, China. Nasal swabs were swirled in $1 \mathrm{~m} l$ PBS, which was then centrifuged at 10, 000 $\mathrm{g}$ for $30 \mathrm{~min}$ to harvest the precipitate for DNA extraction. 
Table 1. Mycoplasma ovipneumoniae strains used for primer design and accession numbers

\begin{tabular}{cccccc}
\hline Strain & GenBank ID & Host & Sample type & Country & Source \\
\hline Y-98 & JOTL00000000 & sheep & Lung & U.S.A. & CVCC $^{\text {a) }}$ \\
SC01 & AFHO00000000 & goat & Lung & China & SWUN \\
TC-1 & JOTE00000000 & goat & Nasal swab & China & SWUN \\
TC-2 & JOTF00000000 & goat & Lung & China & SWUN \\
TC-3 & JOTG00000000 & goat & Nasal swab & China & SWUN \\
TC-4 & JOTH00000000 & goat & Lung & China & SWUN \\
TC-5 & JOTI00000000 & goat & Nasal swab & China & SWUN \\
TC-7 & JOTJ00000000 & goat & Lung & China & SWUN \\
TC-8 & JOTK00000000 & goat & Nasal swab & China & SWUN \\
\hline
\end{tabular}

a) China Veterinary Culture Collection Center; b) Southwest University for Nationalities (our lab collection).

Table 2. Mycoplasmas and other bacterial strains used to test the performance of the developed qPCR for Mycoplasma ovipneumoniae

\begin{tabular}{|c|c|c|c|}
\hline Species & Strain & Source & Real-time PCR \\
\hline \multirow[t]{22}{*}{ M. ovipneumoniae } & Y-98 & $\mathrm{CVCC}^{\mathrm{a})}$ & + \\
\hline & $\mathrm{SC} 01^{\mathrm{e})}$ & $\mathrm{Lab}^{\mathrm{b})}$ & + \\
\hline & $\mathrm{TC}-1^{\mathrm{e})}$ & $\mathrm{Lab}$ & + \\
\hline & $\mathrm{TC}-2^{\mathrm{e})}$ & $\mathrm{Lab}$ & + \\
\hline & $\mathrm{TC}-3^{\mathrm{e})}$ & $\mathrm{Lab}$ & + \\
\hline & TC-4e) & Lab & + \\
\hline & TC-5 ${ }^{\mathrm{e})}$ & $\mathrm{Lab}$ & + \\
\hline & $\mathrm{TC}-7^{\mathrm{e})}$ & Lab & + \\
\hline & TC- $8^{\mathrm{e})}$ & Lab & + \\
\hline & JY1001e) & $\mathrm{Lab}$ & + \\
\hline & $J Y 1002^{e)}$ & Lab & + \\
\hline & $\mathrm{LZ} 1001^{\mathrm{e})}$ & Lab & + \\
\hline & LZ1002e) & $\mathrm{Lab}$ & + \\
\hline & CD1001e) & Lab & + \\
\hline & $\mathrm{CD} 1002^{\mathrm{e})}$ & $\mathrm{Lab}$ & + \\
\hline & QH0145f) & $\mathrm{Lab}$ & + \\
\hline & QH0146 ${ }^{\mathrm{f}}$ & $\mathrm{Lab}$ & + \\
\hline & QH0214 ${ }^{\mathrm{f}}$ & $\mathrm{Lab}$ & + \\
\hline & $\mathrm{QH} 0215^{\mathrm{f})}$ & $\mathrm{Lab}$ & + \\
\hline & GS1001 ${ }^{\mathrm{f})}$ & $\mathrm{Lab}$ & + \\
\hline & GS1002f) & Lab & + \\
\hline & $\mathrm{XJ} 1001^{\mathrm{f})}$ & Lab & + \\
\hline \multirow{2}{*}{ M. capricolum subsp. capripneumoniae } & 87001 & $\mathrm{CVCC}$ & - \\
\hline & 87002 & CVCC & - \\
\hline M. mycoides subsp. mycoides Large Colony & Y-goat & CVCC & - \\
\hline M. putrefaciens & GM1 & ATCC & - \\
\hline M. mycoides subsp. capri & PG3 & $\mathrm{CVCC}$ & - \\
\hline M. agalactiae & PG2 & $\mathrm{CVCC}$ & - \\
\hline M. arginini & G230 & $\mathrm{CVCC}$ & - \\
\hline \multirow[t]{2}{*}{ M. bovis } & $\mathrm{CD}-2$ & $\mathrm{Lab}$ & - \\
\hline & CQ20 & $\mathrm{SWU}^{\mathrm{c})}$ & - \\
\hline \multirow{3}{*}{ M. conjunctivae } & $\mathrm{HRC} / 581 \mathrm{~T}$ & ATCC & - \\
\hline & Mc1001 & $\mathrm{Lab}$ & - \\
\hline & Mc1002 & $\mathrm{Lab}$ & - \\
\hline Mycoplasma capricolum subsp. capricolum & California kid & $\mathrm{Xie}^{\mathrm{d})}$ & - \\
\hline Staphylococcus aureus & ATCC 6538 & $\mathrm{CVCC}$ & - \\
\hline Mannheimia haemolytica & ATCC 31611 & ATCC & - \\
\hline Pasteurella multocida & SC-3 & $\mathrm{Lab}$ & - \\
\hline Escherichia coli & 013 & $\mathrm{Lab}$ & - \\
\hline
\end{tabular}

a) China Veterinary Culture Collection Center; b) Our Laboratory Collection; c) Southwest University, China; d) Genomic DNA, kindly provide by Dr Xiulan Xie at Research Center of Grass and Livestock, Ningxia Academy of Agriculture and Forestry Sciences, China; e) M. ovipneumoniae isolates from goats; f) M. ovipneumoniae isolates from sheep. 
Lung tissues were homogenized in PBS for DNA extraction. Pure DNA was extracted from bacterial cultures, nasal swabs and lung tissues by using a commercial Genomic DNA Isolation Kit (Foregene, Chengdu, China) following the manufacturer's instruction.

All real-time PCRs were performed on an ABI 7300 RealTime PCR Detection System (Applied Biosystems, Foster City, CA, U.S.A.) using SYBR ${ }^{\circledR}$ Premix Ex Taq ${ }^{\mathrm{TM}}$ Kit (Ta$\mathrm{KaRa}$, Otsu, Japan). The $20 \mu \mathrm{l}$ reaction mixture consisted of $10 \mu l$ of SYBR premix, $2 \mu l$ of DNA, $0.5 \mu l$ of each primer $(10 \mu \mathrm{M})$ and $7 \mu l$ of deionized water. The cycling conditions were as follows: $3 \mathrm{~min}$ at $95^{\circ} \mathrm{C}$, followed by 40 cycles of $95^{\circ} \mathrm{C}$ for $30 \mathrm{sec}, 60^{\circ} \mathrm{C}$ for $30 \mathrm{sec}$ and $72^{\circ} \mathrm{C}$ for $30 \mathrm{sec}$ and ending with a melting curve analysis at temperatures ranging from $65^{\circ} \mathrm{C}$ to $95^{\circ} \mathrm{C}$.

In order to rule out the false negative results due to DNA losses during DNA extraction and PCR inhibitor in the template, we used duck enteritis virus DNA as an internal control (IC) [15] to evaluate DNA extraction and PCR efficiency as previously described for other mycoplasmas and IC [10]. Ten $M$. ovipneumoniae broth cultures, 10 nasal swabs and 10 lung tissues were used for this experiment. In brief, duck enteritis virus DNA was added to the lysis buffer L1 (20, 000 DNA copies per $\mathrm{m} l$ of buffer) of the Foregene DNA Isolation Kit prior to DNA extraction. The fixed amount of this internal control had been calculated to yield a mean $\mathrm{C}_{\mathrm{T}}$ value of 27.81 with an SD of 0.86 as calculated from 30 independent runs. qPCR for IC detection was carried out using primers Dev-F 5'-CTCTACGCAGCTTTTGACGATTT-3', Dev-R 5'-AGAAACATACTGTGAGAGTGACGA-3' and TaqMan probe 5'-6-FAM- CCTCCTCCTCGCTGAGTGGCATCCTAMRA-3' as described [15]. The IC was detected in all tested samples with qPCR $\mathrm{C}_{\mathrm{T}}$ values below 29.86, indicating minimal and acceptable DNA losses during DNA extraction and the absence of PCR inhibitors with our qPCR amplification protocol.

To construct a standard curve and determine the detection limit of the assay, M. ovipneumoniae SC01 genomic DNA was used as a quantification standard. The genomic DNA was quantified by measuring the optical density at 260 nm using a DU 800 spectrophotometer (Beckman Coulter, Brea, CA, U.S.A.). The amount of genomic DNA was then calculated based on the genome size $(1,020,601 \mathrm{bp})$. Subsequently, 10-fold serial dilutions of M. ovipneumoniae SC01 genomic DNA were prepared and tested using real-time PCR. Three replicates were tested for each concentration 10 -fold dilution. The genome copy numbers in each reaction and their corresponding $\mathrm{C}_{\mathrm{T}}$ values were used to plot the standard curve (Fig. 1). The detection limit was found to be 22 genomic DNA copies, and a wide linear dynamic range $\left(2.2 \times 10^{2}\right.$ to $2.2 \times 10^{7}$ genomes $)$ was established. The linear correlation between the $\mathrm{C}_{\mathrm{T}}$ values and the logarithm of the DNA copy number was higher than 0.994 .

The serial dilutions were simultaneously tested using a gel-based method based on 16S rDNA gene PCR and a conventional PCR protocol as described [11] to determine the level of sensitivity of our qPCR assay with respect to conventional PCR. Results indicated that our $p 113$ gene qPCR

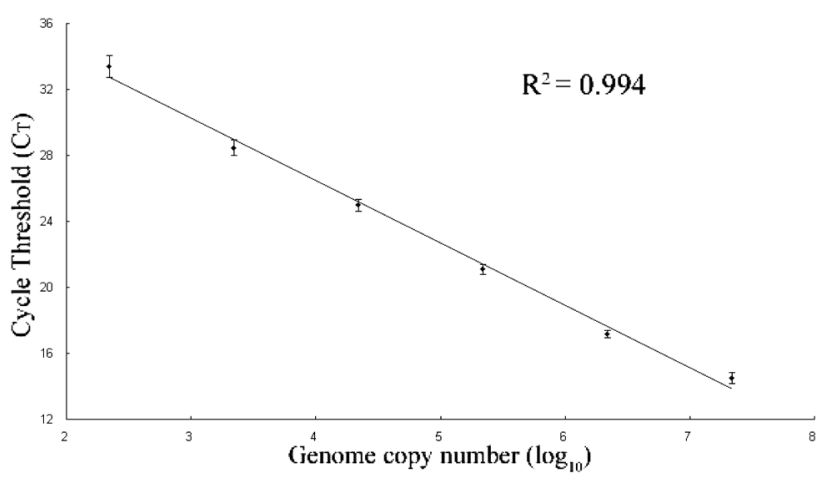

Fig. 1. Correlation between $\mathrm{C}_{\mathrm{T}}$ values and genome copy numbers for M. ovipneumoniae reference strain SC01. Error bars indicate triplicate testing.

was able to detect M.ovipneumoniae three dilutions earlier (1,000 times more sensitive) compared to the gel-based 16S rDNA PCR for which the detection limit was of $2.2 \times 10^{4}$ copies of genomic DNA.

The specificity of the primers $\mathrm{P} 113 \mathrm{~F} / \mathrm{P} 113 \mathrm{R}$ was tested using DNA from 35 mycoplasma strains and 4 bacteria strains listed in Table 2 . The qPCR successfully detected $M$. ovipneumoniae and yielded negative results as expected for the other mycoplasmas and bacteria tested. Melting curve analysis confirmed the presence of a single specific melt peak for each amplicon of $M$. ovipneumoniae strains. The melting temperature was calculated to be $79.6^{\circ} \mathrm{C} \pm 0.4^{\circ} \mathrm{C}$. These results suggested that the qPCR was highly specific for M. ovipneumoniae detection and could therefore be used to discriminate $M$. ovipneumoniae from other bacteria, including Mannheimia haemolytica, Pasteurella multocida and members of the Mycoplasma mycoides cluster, which are causative agents of respiratory illnesses in sheep and goats.

To evaluate the use of qPCR in clinical samples, DNA from 98 nasal swabs and 28 lung samples was tested using both the M. ovipneumoniae qPCR and the conventional $16 \mathrm{~S}$ rDNA PCR assay. The qPCR showed positive results for $59.2 \%$ (58/98) nasal swabs and 75\% (21/28) lung tissues, whereas $16 \mathrm{~S}$ rDNA-based PCR showed a lower rate of positive results (nasal swabs 38.8\%, 38/98; lung tissues $42.9 \%$, 12/28). The qPCR detected M. ovipneumoniae in all samples that tested positive by the $16 \mathrm{~S}$ rDNA PCR assay, indicating good agreement and a higher detection ability with the qPCR for both types of samples (nasal swabs, $P<0.01$; lung tissues, $P<0.05)$. This newly developed qPCR assay might have higher clinical sensitivity than previously described gel-based PCR technique.

To confirm the PCR accuracy and exclude the possibility of false-positive amplifications during qPCR, 10 PCR amplicons ( 5 from nasal swabs and 5 from lungs) were selected, cloned into pMD-T vector (TaKaRa) and sequenced. Sequencing showed that all amplicons were from $M$. ovipneumoniae DNA with a sequence similarity of $95.8-100 \%$ compared to reference strain Y-98. 
Here, we report the development and validation of a qPCR protocol for the detection and quantification of $M$. ovipneumoniae. The quantitative detection of mycoplasmas via $\mathrm{qPCR}$ is valuable, because this approach overcomes the challenges associated with conventional methods. Traditional titration techniques, such as the plate-counting method, which are used for quantitation of bacterial cultures, are impractical for quantitation of mycoplasmas owing to the slow growth and small size of colonies in solid agar surfaces. The qPCR assay presented in this study was shown to be linear over a broad range of $2.2 \times 10^{2}$ to $2.2 \times 10^{7}$ DNA copies with a high correlation coefficient supporting the role of our protocol to accurately quantitate $M$. ovipneumoniae in the samples.

Sensitive detection of mycoplasmas is essential for the diagnosis of mycoplasma infections. The qPCR developed in this study was 1,000 times as sensitive as the existing conventional 16S rDNA-based PCR method. The increased sensitivity of this assay would enable detection of $M$. ovipneumoniae in infected animals with low mycoplasma loads. Although it has been recommended that test sensitivity for conventional PCR can be increased for diagnostic purposes by culture enrichment of clinical specimens [14], such step would not be necessary with a more sensitive qPCR method. Despite the feasibility, enrichment of samples can be cumbersome, because of the fastidious nature and nutritional requirements.

In conclusion, we developed a highly sensitive and rapid SYBR Green-based real-time PCR assay that specifically detects $M$. ovipneumoniae. This assay could be a valuable tool for detection and quantification of M. ovipneumoniae and could have applications in clinical diagnostics and epidemiological studies.

ACKNOWLEDGMENT. This work was supported by the National Nature Science Foundation of China (31272588), the "863" National High-tech Development Research Project (2012AA101304) and the Veterinary Medicine Discipline Program of Southwest University for Nationalities (2014XWD-S0906).

\section{REFERENCES}

1. Becker, C. A. M., Ramos, F., Sellal, E., Moine, S., Poumarat, F. and Tardy, F. 2012. Development of a multiplex real-time PCR for contagious agalactia diagnosis in small ruminants. J. Microbiol. Methods 90: 73-79. [Medline] [CrossRef]

2. Besser, T. E., Cassirer, E. F., Potter, K. A., VanderSchalie, J., Fischer, A., Knowles, D. P., Herndon, D. R., Rurangirwa, F. R., Weiser, G. C. and Srikumaran, S. 2008. Association of Mycoplasma ovipneumoniae infection with population-limiting respiratory disease in free-ranging Rocky Mountain bighorn sheep (Ovis canadensis canadensis). J. Clin. Microbiol. 46: 423-430. [Medline] [CrossRef]

3. Clothier, K. A., Jordan, D. M., Thompson, C. J., Kinyon, J. M., Frana, T. S. and Strait, E. L. 2010. Mycoplasma bovis real-time polymerase chain reaction assay validation and diagnostic performance. J. Vet. Diagn. Invest. 22: 956-960. [Medline] [CrossRef]

4. Dassanayake, R. P., Shanthalingam, S., Herndon, C. N., Subramaniam, R., Lawrence, P. K., Bavananthasivam, J., Cassirer, E. F., Haldorson, G. J., Foreyt, W. J., Rurangirwa, F. R., Knowles, D. P., Besser, T. E. and Srikumaran, S. 2010. Mycoplasma ovipneumoniae can predispose bighorn sheep to fatal Mannheimia haemolytica pneumonia. Vet. Microbiol. 145: 354-359. [Medline] [CrossRef]

5. Edgar, R. C. 2004. MUSCLE: multiple sequence alignment with high accuracy and high throughput. Nucleic Acids Res. 32: 1792-1797. [Medline] [CrossRef]

6. Fitzmaurice, J., Sewell, M., King, C. M., McDougall, S., McDonald, W. L. and O'Keefe, J. S. 2008. A real-time polymerase chain reaction assay for the detection of Mycoplasma agalactiae. N. Z. Vet. J. 56: 233-236. [Medline] [CrossRef]

7. Gorton, T. S., Barnett, M. M., Gull, T., French, R. A., Lu, Z., Kutish, G. F., Adams, L. G. and Geary, S. J. 2005. Development of real-time diagnostic assays specific for Mycoplasma mycoides subspecies mycoides Small Colony. Vet. Microbiol. 111: 51-58. [Medline] [CrossRef]

8. Li, L., Stoeckert, C. J. Jr. and Roos, D. S. 2003. OrthoMCL: identification of ortholog groups for eukaryotic genomes. $\mathrm{Ge}$ nome Res. 13: 2178-2189. [Medline] [CrossRef]

9. Lorenzon, S., Manso-Silván, L. and Thiaucourt, F. 2008. Specific real-time PCR assays for the detection and quantification of Mycoplasma mycoides subsp. mycoides SC and Mycoplasma capricolum subsp. capripneumoniae. Mol. Cell. Probes 22: 324-328. [Medline] [CrossRef]

10. Lorusso, A., Decaro, N., Greco, G., Corrente, M., Fasanella, A. and Buonavoglia, D. 2007. A real-time PCR assay for detection and quantification of Mycoplasma agalactiae DNA. J. Appl. Microbiol. 103: 918-923. [Medline] [CrossRef]

11. McAuliffe, L., Hatchell, F. M., Ayling, R. D., King, A. I. and Nicholas, R. A. 2003. Detection of Mycoplasma ovipneumoniae in Pasteurella-vaccinated sheep flocks with respiratory disease in England. Vet. Rec. 153: 687-688. [Medline] [CrossRef]

12. Ongor, H., Kalin, R. and Acik, M. N. 2011. Detection of $M y$ coplasma ovipneumoniae from goats with nasal discharge by culture and polymerase chain reaction. Pak. Vet. J. 31: 244-248.

13. Rifatbegović, M., Maksimović, Z. and Hulaj, B. 2011. Mycoplasma ovipneumoniae associated with severe respiratory disease in goats. Vet. Rec. 168: 565. [Medline] [CrossRef]

14. Weiser, G. C., Drew, M. L., Cassirer, E. F. and Ward, A. C. 2012. Detection of Mycoplasma ovipneumoniae and M. arginini in bighorn sheep using enrichment culture coupled with genus- and species-specific polymerase chain reaction. J. Wildl. Dis. 48 : 449-453. [Medline] [CrossRef]

15. Yang, F. L., Jia, W. X., Yue, H., Luo, W., Chen, X., Xie, Y., Zen, W. and Yang, W. Q. 2005. Development of quantitative real-time polymerase chain reaction for duck enteritis virus DNA. Avian Dis. 49: 397-400. [Medline] [CrossRef]

16. Yang, F., Tang, C., Wang, Y., Zhang, H. and Yue, H. 2011. Genome sequence of Mycoplasma ovipneumoniae strain SC01. J. Bacteriol. 193: 5018. [Medline] [CrossRef]

17. Yang, F., Zhang, H., Tang, C. and Yue, H. 2013. Sequence analysis and functional prediction of Mycoplasma ovipneumoniae pl13 gene. Hua Nan Nong Ye Da Xue Xue Bao 34: 117-121 (in Chinese). 